Proceeding Series of the Brazilian Society of Computational and Applied Mathematics

\title{
Relação entre Propriedades Micromecânicas de Sistemas Granulares Bidimensionais e Propriedades Geométricas da Tesselação de Voronoi
}

\section{Eduardo Célio Boaventura ${ }^{1}$}

Departamento de Física e Matemática, CEFET-MG, Belo Horizonte, MG, Fernando Andrade Ducha²

Programa de Pós-Graduação em Modelagem Matemática e Computacional - CEFET-MG, Belo Horizonte, MG

\section{Allbens Atman Picardi Faria ${ }^{3}$}

Departamento de Física e Matemática, CEFET-MG, Belo Horizonte, MG,

\begin{abstract}
Resumo. Neste trabalho construímos a tesselação de Voronoi (VT) para dois diferentes tipos de empilhamentos granulares bidimensionais (simulados em computador) sem sobrecarga e sujeitos somente à interação gravitacional. Esses empilhamentos foram formados a partir de dois tipos de deposição: 1) grão-a-grão e 2) tipo chuva. Calculamos, para cada VT, as distribuições estatísticas do número de lados dos polígonos (que representa o número de vizinhos de cada grão) e do tamanho das áreas desses polígonos (que se relaciona com o grau de compactação dos grãos). Através desses dados, buscamos correlacionar as características micromecânicas dessas pilhas granulares, como configurações de forças nas bases, distribuição dos ângulos de contato, número de coordenação etc., com as propriedades geométricas da VT.
\end{abstract}

Palavras-chave. Materiais Granulares, Simulação Computacional, Geometria Computacional, Tesselação de Voronoi.

\section{Introdução}

Materiais granulares apresentam comportamentos diferentes quando submetidos a diferentes formas de perturbação. Podem apresentar características de estado sólido, líquido, gasoso, ou mesmo características próprias não encontradas na grande maioria dos materiais sob um desses estados [4]. Mesmo levando-se em consideração pequenas sutilezas na forma de preparação de tais sistemas, podemos encontrar significantes diferenças nas

\footnotetext{
${ }^{1}$ edboaventura@deii.cefetmg.br

${ }^{2}$ duchaf@dppg.cefetmg.br

3atman@dppg.cefetmg.br
} 
respostas às solicitações externas por eles apresentadas. Podemos exemplificar duas formas diferentes no preparo das amostras devido ao método como elas foram depositadas - grão-a-grão "GG" ou tipo chuva "RL". Outra diferença que podemos impor às nossas amostras é o tipo de perturbação, como, por exemplo, uma força aplicada verticalmente no topo da pilha formada ou uma força aplica em diagonal [1].

Uma ferramenta que tem se mostrado de extrema importância na análise de problemas envolvendo pavimentações no plano euclidiano é a tesselação de Voronoi [6], bem como seu dual, a triangulação de Delaunay. Estas ferramentas nos permitem analisar várias mudanças pautadas em polígonos que cercam os objetos que desejamos estudar. Através da análise das mudanças geométricas, podemos avaliar distâncias entre sítios, áreas de domínio para cada sítio, distribuição estatística das áreas dos polígonos, número de vizinhos de sítios (ou de arestas de polígonos), ângulos de polígonos etc., em função da preparação ou solicitação mecânica.

Estes materiais (granulares) estão presentes na indústria metalúrgica, siderúrgica, de construção, alimentícia etc. Não bastasse esse motivo para estudá-los e compreendê-los podemos citar outro tão importante quanto, que é a geração de conhecimento acadêmico, podendo se transformar em conhecimento aplicado ou não. Atualmente, possuímos uma representativa gama de conhecimentos sobre tais materiais, em fenômenos como: compactação, fluidização, fluxo, formação de arcos, convecção, empuxo reverso, instabilidade de pilhas, biestabilidade, dilatância [8], terremotos, avalanches, oscillons, histerese etc. [5]. Não obstante possuirmos conhecimentos sobre os fenômenos citados, eles estão longe de serem completos e, assim, prosseguimos, então, no estudo de tais sistemas e fenômenos desta vasta área de interesse.

Neste artigo trabalhamos com duas formas de deposição, "grão-a-grão" (GG) e "tipo chuva" (RL), sendo a dinâmica dos grãos, desde o início de sua deposição até a configuração de repouso da pilha, simulada [1] por meio da técnica de Dinâmica Molecular (MD).

Na seção 2 descrevemos o objetivo (ou propósito) deste trabalho e, a seguir, na seção 3 discutimos sobre as ferramentas e o procedimento por nós utilizado. Na seção 4 mostramos os resultados e, na seção 5 fizemos uma discussão sobre esses resultados. Por fim, na seção 6 mostramos as conclusões e perspectivas futuras.

\section{Propósito}

Em 2005, Atman et al [1] analisaram os perfis de resposta às tensões para sistemas granulares sujeitos a preparações distintas. Nesse artigo, discutiu-se sobre medições experimentais em arranjos bidimensionais construídos com grãos fotoelásticos, experimentos em pilhas tridimensionais e, finalmente, apresentaram dados obtidos por meio de simulações numéricas de empilhamentos granulares. Os trabalhos realizados por Atman et al foram executados utilizando-se MD, simulando as trajetórias dos grãos por meio das equações de Newton para o movimento, lançando mão do Método de Runge-Kutta de quarta ordem [7], com um esquema preditor-corretor de Gear.

O propósito de nosso trabalho é mostrar que pode-se chegar às mesmas informações sobre os perfis de resposta às tensões para os sistemas estudados por Atman et al apenas 
utilizando informações geométricas da VT. Nossas informações se pautariam unicamente na análise dos polígonos que formam as tesselações de Voronoi construídas sobre as configurações dos grãos dos sistemas. A hipótese, por nós defendida, é que as respostas das distribuições de forças, número e ângulos de contatos etc., possuem correlação direta com as distribuições geométricas dos polígonos das tesselações de Voronoi.

\section{Métodos}

\subsection{Tesselação (ou diagrama) de Voronoi}

Definimos a distância euclidiana entre dois pontos $p$ e $q$ como sendo dist $(p, q)$. No plano, temos

$$
\operatorname{dist}(p, q):=\sqrt{\left(p_{x}-q_{x}\right)^{2}+\left(p_{y}-q_{y}\right)^{2}}
$$

Seja $P:=\left\{p_{1}, p_{2}, \ldots, P_{n}\right\}$ um conjunto de $n$ pontos distintos no plano; estes pontos são chamados sítios. Nós definimos o diagrama de Voronoi de $P$, Figura 1a, como sendo a subdivisão do plano em $n$ células, uma para cada sítio em $P$, com a propriedade de que um ponto $q$ situa-se na célula correspondente a um sítio $p_{i}$ se, e somente se, $\operatorname{dist}\left(q, p_{i}\right)$ $<\operatorname{dist}\left(q, p_{j}\right)$ para todo $p_{j} \in P$, com $j \neq i$. Denotamos o diagrama de Voronoi de $P$ por $\operatorname{Vor}(P)$. Abusando ligeiramente da terminologia, vamos, às vezes, usar "Vor $(P)$ " ou "diagrama de Voronoi" para indicar apenas as arestas e vértices da subdivisão. Quando dizemos que um diagrama de Voronoi é conexo significa que a união de suas arestas e vértices forma um conjunto conexo. A célula de $\operatorname{Vor}(P)$ que corresponde a um sítio $p_{i}$ é denotada por $v\left(p_{i}\right)$ e nós a chamamos de célula de Voronoi de $p_{i}$ [2]. Em outras palavras, $v\left(p_{i}\right)$ é o polígono que circunscreve o sítio $p_{i}$ ou, ainda, $v\left(p_{i}\right)$ é o conjunto de pontos do plano que estão mais próximos de $p_{i}$ do que de qualquer outro sítio.

\subsection{Procedimento}

Utilizamos as configurações granulares (disposições dos grãos no plano) geradas por diferentes meios dos processos de deposição para construção das tesselações de Voronoi. O centro de cada grão foi considerado como um sítio da tesselação.

Construímos tesselações para dois sistemas diferentes. O primeiro sistema é o "grão-agrão" (GG), deposição na qual apenas um grão por vez cai em posições aleatórias do eixo x; e o segundo, o "tipo chuva" (RL), deposição por meio de vários grãos que caem simultaneamente sobre um substrato. Ambas as deposições ocorrem unicamente sob influência gravitacional. Trabalhamos com cinco amostras de cada sistema. Após a coleta de dados, calculamos as médias e os erros para as distribuições do tamanho das áreas dos polígonos formados e também para as distribuições do número de vizinhos por grão.

Uma maneira simples de construir o diagrama de Voronoi é a seguinte: para cada sítio $p_{i}$, calculamos a interseção comum dos semiplanos $h\left(p_{i}, p_{j}\right)$, Figura $1 \mathrm{~b}$, com $j \neq i$. Desta forma, os algoritmos mais simples gastam $O\left(n^{2} \log n\right)$ para calcular todo o diagrama de Voronoi. Felizmente, pudemos ser mais eficientes, lançamos mão do algoritmo de varredura do plano, comumente chamado de algoritmo de Fortune em homenagem ao seu inventor 
[3]. Com este algoritmo, pudemos calcular todo o diagrama de Voronoi com um tempo $O(n \log n)$, no pior caso.

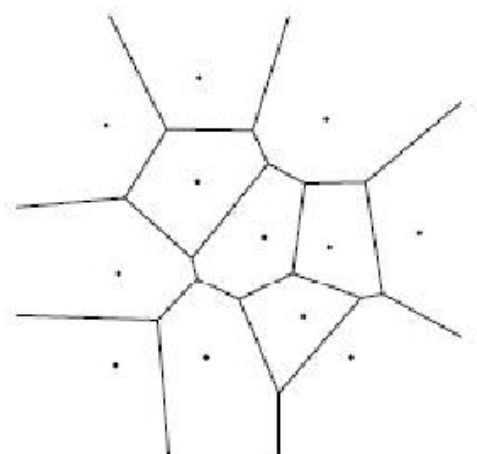

(a)

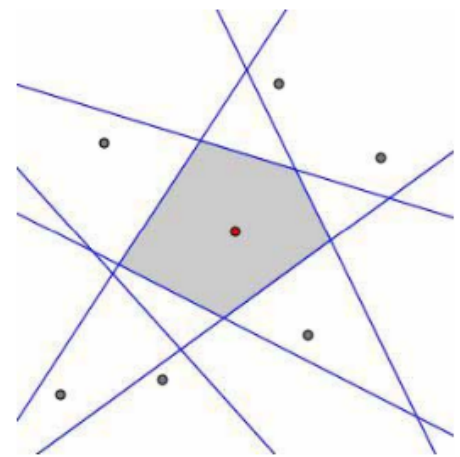

(b)

Figura 1: À esquerda, tesselação de Voronoi e, à direita, semiplanos $h\left(p_{i}, p_{j}\right)[2]$.

\section{Resultados}

Nos trabalhos realizados por Atman et al [1], as simulações baseadas em MD, resolvendo as equações de Newton, mostraram resultados interessantes, tais comos os da Figura 2 .
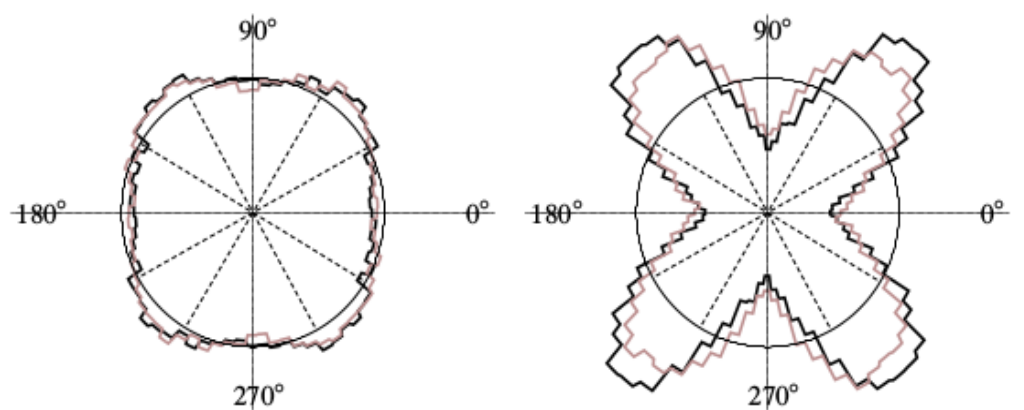

Figura 2: A linha preta se refere aos ângulos de contato enquanto que a linha cinza se refere à orientação das forças. A figura da esquerda representa o sistema RL e a figura da direita o sistema GG. A forma aproximadamente circular do sistema RL indica uma distribuição isotrópica equanto que no sistema GG os ângulos de contato e a orientação das forças são predominantes nos ângulos de $45^{\circ}+n \times 90^{\circ}$, onde $n=0,1,2,3$, demonstrando um caráter anisotrópico. [1].

Os resultados, por nós encontrados, com respeito às distribuições das áreas dos polígonos de Voronoi são mostrados nas Figuras 3 e 4.

Já em relação à quantidade de vizinhos para os grãos, os resultados foram os mostrados na Figura 5. 


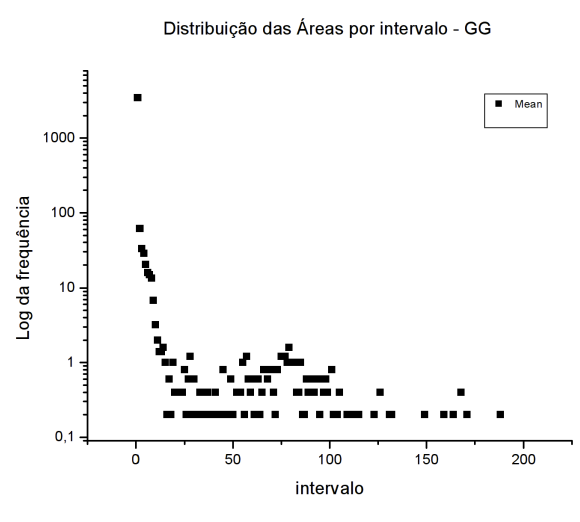

(a) Deposição GG

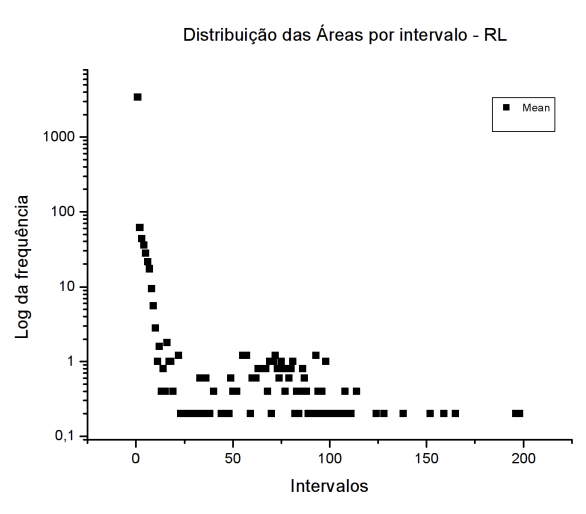

(b) Deposição RL

Figura 3: Distribuição das áreas dos polígonos de Voronoi para pilhas GG (3(a)) e RL(3(b)), sem cargas.

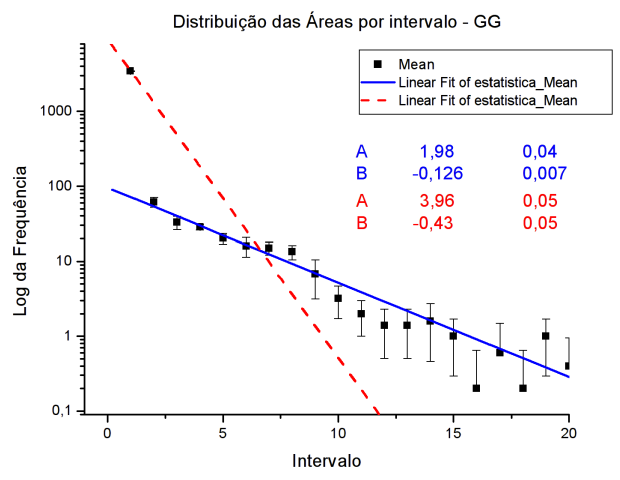

(a) Deposição GG

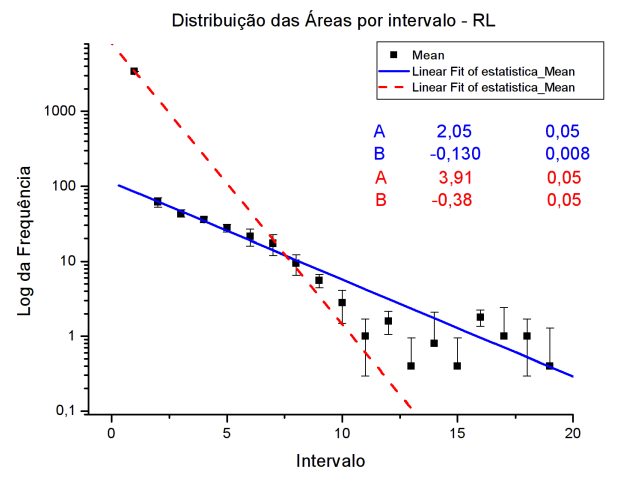

(b) Deposição RL

Figura 4: Distribuição das áreas dos polígonos de Voronoi (até o vigésimo interevalo) para pilhas GG (4(a)) e RL(4(b)), sem cargas.

\section{Discussão dos Resultados}

Os gráficos da Figura 3 foram construídos com 200 intervalos com alcance de 0,0005 unidade de área cada. Para cada intevalo, plotado no eixo $\mathrm{x}$, fizemos a contagem da ocorrencia de áreas a ele pertencente e plotamos o valor do logaritmo desta frequência no eixo y.

Observando a Figura 1a, podemos perceber que, nas bordas, os polígonos são abertos. Dependendo de como é feito o programa, pode-se fechar estes polígonos abertos ligando os pontos mais extremos das arestas das células de Voronoi. Mas, via de regra, estas arestas se fecham em posições relativamente distantes dos sítios que as geram.

Para o nosso caso, esses extremos das arestas abertas superam em muito as posições dos últimos grãos das pilhas. Obviamente, as áreas desses polígonos extremos serão muito 


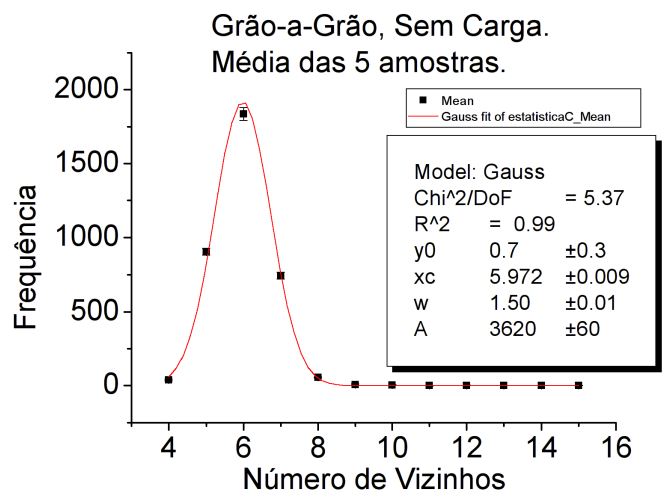

(a) Deposição GG

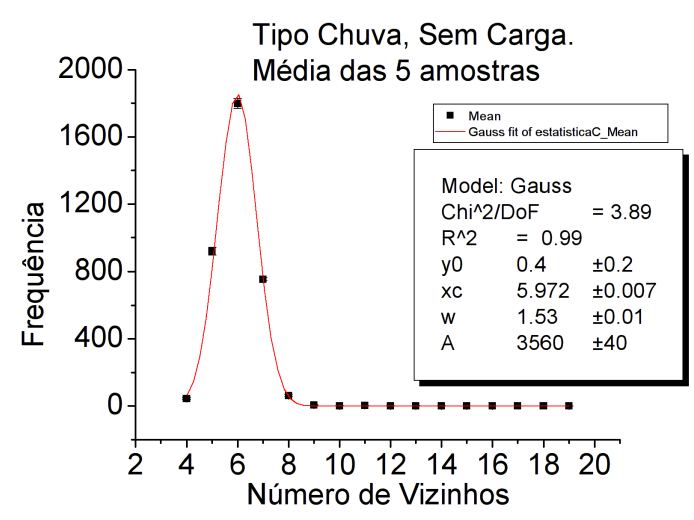

(b) Deposição RL

Figura 5: Distribuição do número de vizinhos por grão para pilhas sem cargas, ou seja, sujeitas somente ao próprio peso.

maiores do que as áreas dos polígonos imersos no interior da tesselação, que são por natureza bem delimitados. Isto justifica o ajuste apenas da parte inicial dos dados.

Sendo assim, fizemos um ajuste linear (linha contínua) considerando somente os 20 primeiros intervalos, Figura 4, desprezando o primeiro (intervalo), que se mostrou uma frequência dominante, e os demais, com áreas relativamente grandes. Logo em seguida, fizemos um ajuste (linha tracejada) considerando os 20 primeiros intervalos, porém, desta vez considerando o primeiro intervalo, ou seja, considerando, também, o dominante.

\section{Conclusões}

Os gráficos para as distribuições de áreas mostram que há uma predominância das áreas menores em ambos os sistemas, GG e RL. Ambas as distribuições, Figuras 3 e 4, seguem um decaimento exponencial e este resultado, até onde apuramos, é inédito na literatura.

Considerando os primeiros 20 intervalos, excluindo o primeiro, ou dominante, não conseguimos uma distinção clara entre os dois sistemas, $\log y=-0,126 x+1,98$ para o GG e $\log y=-0,130 x+2,05$ para o RL. Uma diferença de inclinação no ajuste, que classificamos como inconclusiva, de $3 \%$.

Podemos observar uma sutil diferença entre as inclinações dos ajustes quando consideramos o intevalo dominante. Para o sistema GG obtivemos $\log y=-0,43 x+3,96$ e para o sistema RL obtivemos $\log y=-0,38 x+3,91$, ou seja, o sistema GG exibe uma inclinação $13 \%$ maior, em módulo, do que o sistema RL. Isto indica que o sistema GG concentra áreas ainda menores do que o sistema RL, em outras palavras, o sistema GG é mais regular. Faremos, posteriormente, uma análise pormenorizada entre os intervalos dominantes.

O número de vizinhos por grão, Figura 5, seguiu uma distribuição gaussiana para os dois casos (GG e RL), e não houve uma distinção aparente entre eles. 
Para os dois sistemas houve uma predominância de polígonos hexagonais, pentagonais e heptagonais, respectivamente. Estes polígonos representam a quase totalidade das ocorrências.

Os resultados aqui apresentados ainda são preliminares e os estudos sobre as propriedades dos sistemas em questão continuam em desenvolvimento. Pretendemos, a seguir, construir as tesselações de Voronoi para sistemas GG e RL com cargas aplicadas, e dos quais a rede de forças devido à interação gravitacional tenha sido subtraída, de modo que possamos observar se há distinções nas distribuições das áreas e do número de lados dos polígonos de Voronoi, relacionando estas distinções com as funções resposta (perfis de forças nas bases das pilhas granulares) e com a propriedade intrinseca do sistema de se mostrar isotrópico ou anisotrópico.

Pretendemos também construir a triangulação de Delaunay (estrutura dual à VT) para esses sistemas e verificar se podemos obter as mesmas respostas fornecidas pela tesselação de Voronoi.

\section{Agradecimentos}

Agradeço a todos que contribuíram ao conhecimento necessário à elaboração deste artigo e, também, ao CEFET-MG, pela oportunidade de dedicação exclusiva a este estudo.

\section{Referências}

[1] A. P. F. Atman, P. Brunet, J. Geng, G. Reydellet, G. Combe, P. Claudin, R. P. Behringer and E. Clément, Sensitivity of the stress response function to packing preparation, J. Phys.: Condens. Matter, vol. 17 (2005) S2391-S2403.

[2] M. de Berg, O Cheong, M. V. Kreveld and M. Overmars, Computational Geometry: Algorithms and and Applications, Third Edition, Springer, Cap. 7, (2008).

[3] A. Fortune, A sweepline algorithm for voronoi diagrams, Algorithmica, Springer, vol. 2, n. 1-4, p.153-174, (1987).

[4] M. H. Jaeger and S. R. B. R. P. Nagel, Granular solids, liquids, and gases. Reviews of Modern Physics, v. 68, n. 4, p. 1259, 1996.

[5] A. Mehta, Granular physics. 1. ed. Cambridge: Cambridge University Press, 2007.

[6] A. Okabe and B. S. K. C. S. N. Boots, Spatial tessellations: concepts and applications of Voronoi diagrams. 2. ed. Chichester: John Wiley \& Sons, 2009.

[7] T. Pang, An Introduction to Computational Physics, Second Edition, Cambridge University Press, Cap. 4, (2006).

[8] O. Reynolds, On the dilatancy of media composed of rigid particles in contact. with experimental illustrations. The London, Edinburgh, and Dublin Philosophical Magazine and Journal of Science, v. 20, n. 127, p. 469-481, 1885. 